OPEN ACCESS

Edited by:

Mingtao Zeng,

Texas Tech University Health Sciences

Center El Paso, United States

Reviewed by:

Tamar Ben-Yedidia,

BiondVax Pharmaceuticals Ltd., Israel Marc Paul Girard,

Université Paris Diderot, France

*Correspondence:

Ye Shen

yeshen@uga.edu

Specialty section

This article was submitted to Vaccines and Molecular Therapeutics, a section of the journal

Frontiers in Immunology

Received: 16 December 2020 Accepted: 08 February 2021

Published: 03 March 2021

Citation:

Sung M-H, Shen Y, Handel A, Bahl J and Ross TM (2021) Longitudinal Assessment of Immune Responses to

Repeated Annual Influenza

Vaccination in a Human Cohort of Adults and Teenagers.

Front. Immunol. 12:642791.

doi: 10.3389/fimmu.2021.642791

\section{Longitudinal Assessment of Immune Responses to Repeated Annual Influenza Vaccination in a Human Cohort of Adults and Teenagers}

\author{
Meng-Hsuan Sung ${ }^{1}$, Ye Shen ${ }^{1 *}$, Andreas Handel ${ }^{1,2,3}$, Justin Bahl ${ }^{1,3,4,5}$ and Ted M. Ross ${ }^{4,5}$ \\ ${ }^{1}$ Department of Epidemiology and Biostatistics, College of Public Health, University of Georgia, Athens, GA, United States, \\ ${ }^{2}$ College of Public Health, Health Informatics Institute, University of Georgia, Athens, GA, United States, ${ }^{3}$ Center for the \\ Ecology of Infectious Diseases, University of Georgia, Athens, GA, United States, ${ }^{4}$ Department of Infectious Diseases, \\ College of Veterinary Medicine, University of Georgia, Athens, GA, United States, ${ }^{5}$ Center for Vaccines and Immunology, \\ University of Georgia, Athens, GA, United States
}

Background: The overall performance of a multiple component vaccine assessed by the vaccine-elicited immune responses across various strains in a repeated vaccination setting has not been well-studied, and the comparison between adults and teenagers is yet to be made.

Methods: A human cohort study was conducted at the University of Georgia, with 140 subjects (86 adults and 54 teenagers) repeatedly vaccinated in the 2017/2018 and 2018/2019 influenza seasons. Host information was prospectively collected, and serum samples were collected before and after vaccination in each season. The association between host factors and repeated measures of hemagglutination inhibition ( $\mathrm{HAl}$ ) composite scores was assessed by generalized linear models with generalized estimating equations.

Results: The mean HAl composite scores for the entire sample ( $t=4.26, d f=139, p$ $<0.001)$ and the teenager group $(t=6.44, d f=53, p<0.001)$ declined in the second season, while the changes in the adults were not statistically significant ( $t=-1.14$, $d f=85, p=0.26$ ). A mixture pattern of changes in both directions was observed in the adults when stratified by prior vaccination. In addition, the regression analysis suggested an interactive effect of age and BMI on the HAl composite scores in the overall population (beta $=0.005 ; 95 \% \mathrm{Cl}, 0.0008-0.01$ ) and the adults (beta $=0.005 ; 95 \%$ $\mathrm{Cl}, 0.0005-0.01)$.

Conclusions: Our study found distinct vaccine-elicited immune responses between adults and teenagers when both were repeatedly vaccinated in consecutive years. An interactive effect of age and BMI on the HAl composite scores were identified in the overall population and the adults.

Keywords: influenza vaccine, hemagglutination inhibition assay, composite score, repeat vaccination, immune responses 


\section{INTRODUCTION}

While there is general agreement that influenza virus vaccines provide some protection against infection, the impact that repeated annual influenza virus vaccination has on the level of protection is still not fully understood (1-5). Hoskins et al. concluded that repeated vaccination did not provide a long-term benefit in reducing the cumulative attack rate when compared with no vaccination and single vaccination (1). However, Keitel et al. reported a contradicting finding showing that repeated annual vaccination resulted in better vaccine efficacy than a single administration (2). Both studies found that subjects' pre-vaccination hemagglutination inhibition (HAI) titers and previous vaccination status significantly influenced their postvaccination HAI titers $(3,4)$. Others observed similar levels of vaccine effectiveness among those undergoing vaccination in the current season only and those repeatedly vaccinated in consecutive seasons, implying that repeated vaccination may not have impaired antibody responses (6).

The impact of host factors on the strength of vaccine-elicited immune responses have been widely studied. Several studies showed that aging has an effect on serological responses (79). The impact of age on immune response may interact with individual vaccination and infection histories $(7,9)$. Females had significantly higher antibody titers than males regardless of the influenza strain and dosage of the vaccine (10-12). Interactions between age and sex were also reported, with greater sex differences observed in older adults (13). Obesity is also an important factor correlating with human antibody responses to the influenza vaccine. Several studies found significantly higher initial serologic responses to $\mathrm{H} 1 \mathrm{~N} 1$ vaccines among obese adults compared to adults of normal weights $(14,15)$. However, more significant declines in influenza antibody titers and CD8+ Tcell activation post-vaccination were observed among obese participants, indicating the delayed or blunted immune responses against H1N1 in the obese hosts (16).

While sophisticated statistical models have been used in some cohort studies to account for potential within-person dependency $(17,18)$, only a small fraction of the published studies considered the correlation among the repeatedly measured antibody responses from the same subject. Influenza virus vaccines are often evaluated by the antibody response they elicit, particularly the hemagglutination inhibition (HAI) titers. Current guidelines often evaluate influenza vaccines by each single vaccine component, and HAI titers of 1:40 or greater are considered to be associated with clinical protection (1921). However, the immune response for a single strain cannot evaluate the overall performance of a multiple component vaccine in consecutive years, given the potential changes of the subtype strains included in the vaccine. To study the overall vaccine-elicited antibody responses from multiple influenza strains, an HAI composite score was proposed to evaluate the serological changes in all 4 vaccine components (22). In this study, we examined the longitudinal changes in the vaccine-elicited immune responses and their associations with host factors in a human cohort with both adults and teenagers.

\section{METHODS}

\section{Study Design}

A human cohort vaccine study was initiated in the 20162017 influenza season and has been recruiting subjects annually from the Athens, GA, USA metropolitan region in subsequent years. Human sera samples and individual information were collected and tested at the University of Georgia. From 2017-2018 through 2018-2019, eligible subjects, including teenagers (12-18 years) and adults ( $\geq 18$ years) who had not yet received the seasonal influenza vaccine, were enrolled beginning in September of each year. The details of vaccine formulation and immune response assays have been previously published (22). The influenza strains included in the vaccine formulation for the 2017-2018 season were: A/ Michigan/2015 (H1N1), A/Hong Kong/2014 (H3N2), B/Phuket/2013 (Yamagata-lineage), and B/Brisbane/2008 (Victoria-lineage). In the 2018-2019 season, the vaccine formulation changed to: A/ Michigan/2015 (H1N1), A/ Singapore/2016 (H3N2), B/Phuket/2013 (Yamagata-lineage), and $\mathrm{B} /$ Colorado/2017 (Victoria-lineage).

\section{Data Collections}

Participants who received the standard dose split-virion (IIV) version of licensed FluzoneTM (Sanofi Pasteur, Swiftwater, PA, USA) were selected in this study, with 255 and 242 subjects in the 2017-2018 season and 2018-2019 season, respectively (Supplementary Table 1). Within the 2 consecutive flu seasons, subjects who re-enrolled in the second year would have repeated measurements of immune responses. A total of 140 participants who had assessments of immune responses in both seasons were included in the main longitudinal analysis.

In each influenza season, HAI activity was tested on collected serum samples at Day 0 prior to vaccination and then at Day $21 / 28$ post-vaccination. In addition, individual-level host factors data were prospectively collected. Information on age, sex, race, Body Mass Index (BMI), comorbidity, the month of vaccination, and prior vaccination were collected at first visiting of each year for every participant. The ages of subjects were measured in years. Race included White, Black, American Indian, Asian, and Hispanic. Since over $80 \%$ of subjects were Whites, the race variable was eventually dichotomized into White vs. nonWhite for the subsequent modeling analysis. BMI was calculated by a person's weight in kilograms divided by the square of height in meters. Comorbidity information was self-reported by the participants in each year at enrollment (including diabetes, asthma, hypertension, depression, anxiety, glaucoma, and other uncommon conditions). Day 0 of the 2017-2018 season was considered as our study baseline, and prior vaccination was determined by whether an individual received a flu shot in the 2016-2017 flu season.

\section{Statistical Analysis}

An HAI composite score was created by summing the increasing folds of changes in HAI titers among the 4 influenza strains included in the vaccine formulation in a given year (22). The score was considered as the major outcome assessing overall 
vaccine-elicited immune responses and was calculated as

$$
\begin{aligned}
f(H A I) & =\left(\log _{2} \frac{H A I_{\text {post }}}{H A I_{\text {pre }}}\right)_{H 1 N 1}+\left(\log _{2} \frac{H A I_{\text {post }}}{H A I_{\text {pre }}}\right)_{H 3 N} \\
& +\left(\log _{2} \frac{H A I_{\text {post }}}{H A I_{\text {pre }}}\right)_{\text {Bvic }}+\left(\log _{2} \frac{H A I_{\text {post }}}{H A I_{\text {pre }}}\right)_{\text {Byam }}^{,},
\end{aligned}
$$

where $\mathbf{H A I}_{\text {pre }}$ and $\mathbf{H A I}$ post are HAI titers measured pre- and post- vaccination.

Descriptive statistics on all study participants' demographics were obtained for each flu season. Further, the descriptive statistics for re-enrolled subjects were stratified by flu seasons and age groups (adults vs. teenagers). Box plots were generated for the general population and each age group, stratified by previous vaccination status. Generalized linear models (GLM) with generalized estimating equations (GEE) were then applied to model the associations between the repeated measurements of HAI composite scores and host factors. Covariates included in the model were baseline HAI titers (geometric mean of 4 strains' baseline HAI titers), age, sex, BMI, comorbidity, years (flu season), and prior vaccination history. When included in the regression models, comorbidity was dichotomized by whether an individual possessed at least one comorbidity condition. The final model used the repeatedly measured HAI scores as the outcome and included all covariates and the interaction terms between age and sex, and age and BMI. The compound symmetry correlation structure was adopted in GEE to account for the correlations of repeated measurements from the same subject. In a separate analysis, subjects with HAI composite scores above and below 4 were defined as responders and non-responders, respectively (22). The dichotomized outcome was subsequently modeled by a logistic regression with GEE following the same procedure as the linear model. Subgroup analyses stratified by age were performed by splitting the cohort population into teenagers and adults by a threshold of 18 years old during the 2017-2018 season. The same model settings and scenarios as the overall analysis were then repeated. Additional stratified analyses were conducted in various settings to assess the heterogeneity among different age groups in adults, and to compare repeatedly vaccinated teenagers with those newly enrolled in 2018-2019. All statistical analyses were performed using R software version 4.0.2 (23) with a significance level set at $P=0.05$.

\section{Ethics Statement}

Study participants were recruited at the UGA Clinical Trials Research Unit (CTRU) in Athens, GA, USA, and enrolled with written, informed consent. The Institutional Review Board of the University of Georgia reviewed and approved the study procedures, informed consent, and data collection documents.

\section{RESULTS}

\section{Descriptive Analysis}

Descriptive statistics on all enrolled participants in our cohort study during the period of 2017-2018 to 2018-2019 are presented in Supplementary Table 1). Overall, white participants contributed over $80 \%$ of the total sample size. A noticeable change was that the peak month of receiving vaccination shifted from November of 2017 to September/October of 2018.

Characteristics of the 140 subjects used for our main analyses are presented in Table 1. These were participants who were enrolled in the study in both 2017-2018 and 2018-2019 seasons, and thus considered the true cohort repeatedly vaccinated and assessed in both years. Racial composition and patterns of peak vaccination month were similar to the total sample. There were 86 adults and 54 teenagers, respectively. Characteristics were stratified and compared between the 2 groups. Adults had higher

\begin{tabular}{|c|c|c|c|c|c|c|}
\hline & \multicolumn{3}{|c|}{ 2017-2018 } & \multicolumn{3}{|c|}{ 2018-2019 } \\
\hline & Overall & Adults & Teenagers & Overall & Adults & Teenagers \\
\hline Sample sizes & 140 & 86 & 54 & 140 & 86 & 54 \\
\hline Age & $\begin{array}{c}29.26 \\
(17.86)\end{array}$ & $\begin{array}{c}38.73 \\
(16.88)\end{array}$ & $\begin{array}{l}14.17 \\
(1.24)\end{array}$ & $\begin{array}{c}30.21 \\
(17.73)\end{array}$ & $\begin{array}{c}39.67 \\
(16.66)\end{array}$ & $\begin{array}{l}15.13 \\
(1.29)\end{array}$ \\
\hline $\begin{array}{l}\text { Adults } \\
\text { ( } \geq 18 \text { years) }\end{array}$ & $\begin{array}{c}86 \\
(61.4 \%)\end{array}$ & & & $\begin{array}{c}86 \\
(61.4 \%)\end{array}$ & & \\
\hline $\begin{array}{l}\text { Teenagers } \\
\text { (<18 years) }\end{array}$ & $\begin{array}{c}54 \\
(38.6 \%)\end{array}$ & & & $\begin{array}{c}54 \\
(38.6 \%)\end{array}$ & & \\
\hline BMl & $\begin{array}{l}25.46 \\
(5.62)\end{array}$ & $\begin{array}{l}27.91 \\
(5.41)\end{array}$ & $\begin{array}{l}21.58 \\
(3.26)\end{array}$ & $\begin{array}{l}25.66 \\
(5.56)\end{array}$ & $\begin{array}{l}27.86 \\
(5.46)\end{array}$ & $\begin{array}{l}22.16 \\
(3.61)\end{array}$ \\
\hline $\begin{array}{l}\text { Sex } \\
\text { (Male) }\end{array}$ & $\begin{array}{c}57 \\
(40.7 \%)\end{array}$ & $\begin{array}{c}34 \\
(39.5 \%)\end{array}$ & $\begin{array}{c}23 \\
(42.6 \%)\end{array}$ & $\begin{array}{c}57 \\
(40.7 \%)\end{array}$ & $\begin{array}{c}34 \\
(39.5 \%)\end{array}$ & $\begin{array}{c}23 \\
(42.6 \%)\end{array}$ \\
\hline \multicolumn{7}{|l|}{ Race } \\
\hline White & $\begin{array}{c}114 \\
(81.4 \%)\end{array}$ & $\begin{array}{c}69 \\
(80.2 \%)\end{array}$ & $\begin{array}{c}45 \\
(83.3 \%)\end{array}$ & $\begin{array}{c}114 \\
(81.4 \%)\end{array}$ & $\begin{array}{c}69 \\
(80.2 \%)\end{array}$ & $\begin{array}{c}45 \\
(83.3 \%)\end{array}$ \\
\hline $\begin{array}{l}\text { African } \\
\text { American }\end{array}$ & $\begin{array}{c}6 \\
(4.3 \%)\end{array}$ & $\begin{array}{c}5 \\
(5.8 \%)\end{array}$ & $\begin{array}{c}1 \\
(1.9 \%)\end{array}$ & $\begin{array}{c}6 \\
(4.3 \%)\end{array}$ & $\begin{array}{c}5 \\
(5.8 \%)\end{array}$ & $\begin{array}{c}1 \\
(1.9 \%)\end{array}$ \\
\hline Other & $\begin{array}{c}20 \\
(14.3 \%)\end{array}$ & $\begin{array}{c}12 \\
(14 \%)\end{array}$ & $\begin{array}{c}8 \\
(14.8 \%)\end{array}$ & $\begin{array}{c}20 \\
(14.3 \%)\end{array}$ & $\begin{array}{c}12 \\
(14 \%)\end{array}$ & $\begin{array}{c}8 \\
(14.8 \%)\end{array}$ \\
\hline $\begin{array}{l}\text { Comorbidity } \\
\text { (Yes) }\end{array}$ & $\begin{array}{c}31 \\
(22.1 \%)\end{array}$ & $\begin{array}{c}24 \\
(27.9 \%)\end{array}$ & $\begin{array}{c}7 \\
(13 \%)\end{array}$ & $\begin{array}{c}36 \\
(25.7 \%)\end{array}$ & $\begin{array}{c}22 \\
(25.6 \%)\end{array}$ & $\begin{array}{c}14 \\
(25.9 \%)\end{array}$ \\
\hline \multicolumn{7}{|c|}{ Month of vaccination } \\
\hline September & $\begin{array}{c}11 \\
(7.9 \%)\end{array}$ & $\begin{array}{c}11 \\
(12.8 \%)\end{array}$ & $\begin{array}{c}0 \\
(0 \%)\end{array}$ & $\begin{array}{c}61 \\
(43.6 \%)\end{array}$ & $\begin{array}{c}44 \\
(51.2 \%)\end{array}$ & $\begin{array}{c}17 \\
(31.5 \%)\end{array}$ \\
\hline October & $\begin{array}{c}36 \\
(25.7 \%)\end{array}$ & $\begin{array}{c}27 \\
(31.4 \%)\end{array}$ & $\begin{array}{c}9 \\
(16.7 \%)\end{array}$ & $\begin{array}{c}55 \\
(39.3 \%)\end{array}$ & $\begin{array}{c}31 \\
(36 \%)\end{array}$ & $\begin{array}{c}24 \\
(44.4 \%)\end{array}$ \\
\hline November & $\begin{array}{c}64 \\
(45.7 \%)\end{array}$ & $\begin{array}{c}30 \\
(34.9 \%)\end{array}$ & $\begin{array}{c}34 \\
(63 \%)\end{array}$ & $\begin{array}{c}21 \\
(15 \%)\end{array}$ & $\begin{array}{c}9 \\
(10.5 \%)\end{array}$ & $\begin{array}{c}12 \\
(22.2 \%)\end{array}$ \\
\hline December & $\begin{array}{c}12 \\
(8.6 \%)\end{array}$ & $\begin{array}{c}7 \\
(8.1 \%)\end{array}$ & $\begin{array}{c}5 \\
(9.3 \%)\end{array}$ & $\begin{array}{c}1 \\
(0.7 \%)\end{array}$ & $\begin{array}{c}1 \\
(1.2 \%)\end{array}$ & $\begin{array}{c}0 \\
(0 \%)\end{array}$ \\
\hline January & $\begin{array}{c}14 \\
(10 \%)\end{array}$ & $\begin{array}{c}11 \\
(12.8 \%)\end{array}$ & $\begin{array}{c}3 \\
(5.5 \%)\end{array}$ & $\begin{array}{c}2 \\
(1.4 \%)\end{array}$ & $\begin{array}{c}1 \\
(1.2 \%)\end{array}$ & $\begin{array}{c}1 \\
(1.9 \%)\end{array}$ \\
\hline February & $\begin{array}{c}3 \\
(2.1 \%)\end{array}$ & $\begin{array}{c}0 \\
(0 \%)\end{array}$ & $\begin{array}{c}3 \\
(5.5 \%)\end{array}$ & $\begin{array}{c}0 \\
(0 \%)\end{array}$ & $\begin{array}{c}0 \\
(0 \%)\end{array}$ & $\begin{array}{c}0 \\
(0 \%)\end{array}$ \\
\hline March & $\begin{array}{c}0 \\
(0 \%)\end{array}$ & $\begin{array}{c}0 \\
(0 \%)\end{array}$ & $\begin{array}{c}0 \\
(0 \%)\end{array}$ & $\begin{array}{c}0 \\
(0 \%)\end{array}$ & $\begin{array}{c}0 \\
(0 \%)\end{array}$ & $\begin{array}{c}0 \\
(0 \%)\end{array}$ \\
\hline $\begin{array}{l}\text { Last season } \\
\text { vaccination } \\
\text { (Yes) }\end{array}$ & $\begin{array}{c}106 \\
(75.7 \%)\end{array}$ & $\begin{array}{c}78 \\
(90.7 \%)\end{array}$ & $\begin{array}{c}28 \\
(51.9 \%)\end{array}$ & $\begin{array}{c}140 \\
(100 \%)\end{array}$ & $\begin{array}{c}86 \\
(100 \%)\end{array}$ & $\begin{array}{c}54 \\
(100 \%)\end{array}$ \\
\hline $\begin{array}{l}\text { HAl } \\
\text { composite } \\
\text { score }\end{array}$ & $\begin{array}{c}4.99 \\
(5.62)\end{array}$ & $\begin{array}{l}2.58 \\
(2.7)\end{array}$ & $\begin{array}{l}8.81 \\
(6.84)\end{array}$ & $\begin{array}{l}2.88 \\
(2.26)\end{array}$ & $\begin{array}{l}2.97 \\
(2.33)\end{array}$ & $\begin{array}{l}2.74 \\
(2.17)\end{array}$ \\
\hline
\end{tabular}

TABLE 1 | Descriptive statistics of subjects re-enrolled in both seasons.

Standard deviations/percentages in parentheses for continuous/categorical variables. 
BMIs and were more likely to be vaccinated during the previous flu season than the teenagers. A decline in the mean HAI composite score was observed from year 1 (2017-2018) to year 2 (2018-2019). This change was mainly driven by a decline in the teenage group (Figure 1). Their individual-level trajectories of changes were also quite distinct (Figure 2), with a mixture pattern of changes in both directions observed in the adults and a downward trend dominating the teenagers. The decline was also more apparent in those who were not vaccinated in 20162017. A subgroup analysis between younger adults (age $<50$ ) and older adults (age $\geq 50$ ) was further considered, and we noticed a difference in average BMI between younger adults and older adults (Supplementary Table 2).

\section{Assessment of Changes}

Box plots of HAI composite scores for the total sample and each age subgroups were also shown in Figure 1. Paired T-tests were performed to detect the differences in the mean HAI composite scores from year 1 to year 2 . The scores for the total sample $(t=4.26, d f=139, p<0.001)$ and the teenagers group $(t=$ $6.44, d f=53, p<0.001)$ were significantly decreased in the second year, while the changes in the adults were not statistically significant $(t=-1.14, d f=85, p=0.26)$. When stratified by prior vaccination (Figure 2), significant declines in the second year were observed in participants without prior vaccination $(t=6.78, d f=33, p<0.001)$, but not in those with prior vaccination $(t=0.70, d f=105, p=0.49)$. The mean HAI composite scores of the adults increased in those with prior vaccination $(t=-2.56, d f=77, p=0.01)$ but decreased in those without previous vaccination $(t=2.61, d f=7, p=$ $0.04)$. Meanwhile, significant declines were observed in teenagers regardless of their vaccine history (with prior vaccination: $t=$ 3.01, $d f=27, p=0.006$; without prior vaccination: $t=6.70$, $d f=25, p<0.001)$. We further compared the yearly mean fold changes of HAI titers (log2 scale) in each influenza virus strain included in the 2 years (Figure 3). The average folds changes of Michigan/2015 (H1N1) $(t=4.43, d f=139, p<$ 0.001 ) and Phuket/2013 (Yamagata) $(t=3.26, d f=139, p$ $=0.001$ ), two strains included in both years' vaccine formula, declined significantly in the second year. For H3N2 where the strains included in the vaccine for each flu season had a change, no statistically significant differences were detected for Hong Kong/2014 (H3N2) $(t=1.809, d f=139, p=0.07)$ and Singapore/2016 (H3N2) $(t=1.15, d f=139, p=0.25)$ in terms of yearly fold changes of HAI titers between the consecutive years. The influenza B/Victoria strain in the vaccine changed between the 2 years as well. Brisbane/2008 (Victoria) $(t=3.88$, $d f=139, p<0.001$ ) had significantly different annual fold changes between $17 / 18$ and $18 / 19$, but no such difference was observed for Colorado/2017 (Victoria) $(t=-0.05, d f=139, p=$ 0.96). We further explored these changes stratified by age group and prior vaccination status (Figures 4A,B). An overall decline trend was observed in teenagers regardless of their previous year vaccination, and such declines were statistically significant across all strains for those vaccinated in 2016-2017. For adults with prior vaccination, the changes were mostly insignificant, with significant declines observed in Michigan/2015 (H1N1) and Singapore/2016 (H3N2). Interestingly, we also noticed an increasing trend of such fold changes in most strains among adults vaccinated in 2016-2017. These results were generally consistent with the overall changes in the HAI composite scores shown in Figure 2.

Stratified analyses in teenagers with and without prior vaccination revealed that among the teenagers (16 subjects) who shad a much higher level of antibody responses to the 2017-2018 vaccination than the rest of group, 12 of them did not receive influenza vaccination in the 2016-2017 flu season (Supplementary Figure 1). This pattern is consistent with the results in Figure 2 showing that teenagers without prior vaccination had higher HAI composite scores in 2017-2018. The

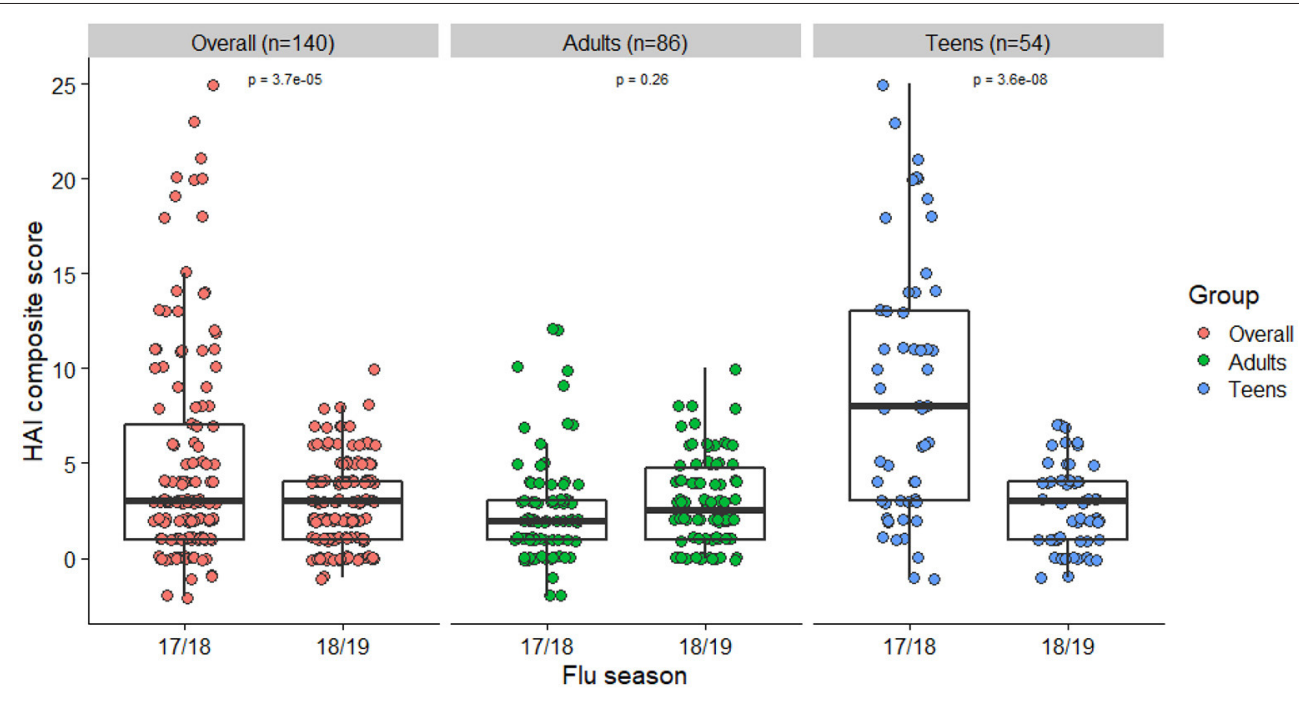

FIGURE 1 | HAI composite scores among different age groups in $17 / 18$ and $18 / 19(N=140)$. 

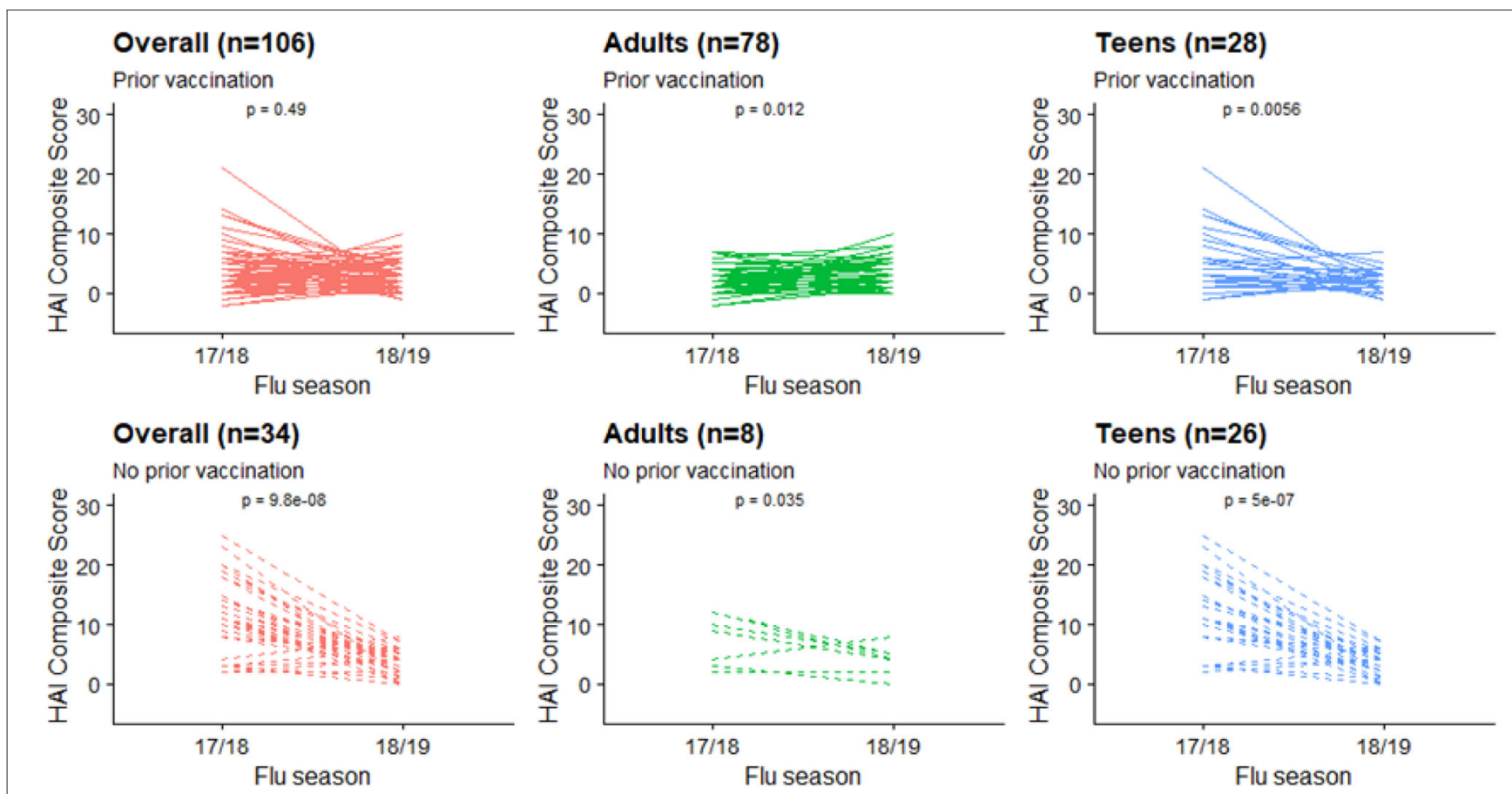

FIGURE 2 | HAI composite scores changes at individual level, stratified by age groups and prior vaccination status.

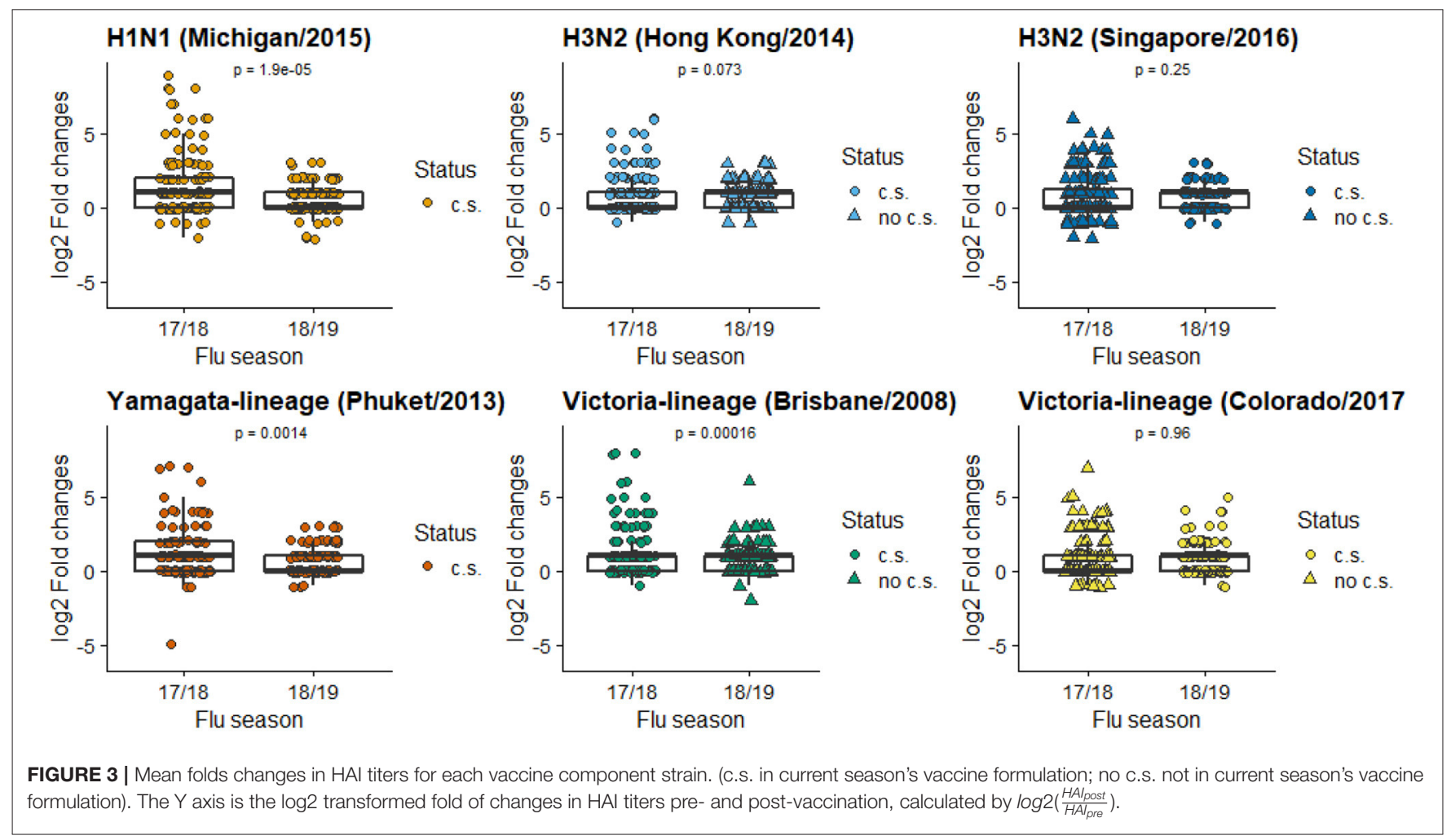

same pattern was not observed in the subsequent year where every participant was vaccinated in the previous season. To better understand the potential impact of repeated vaccination in teenagers, we conducted an additional analysis to include teenagers who participated in the 2018-2019 season but were not enrolled in the 2017-2018 season. Supplementary Table 5 


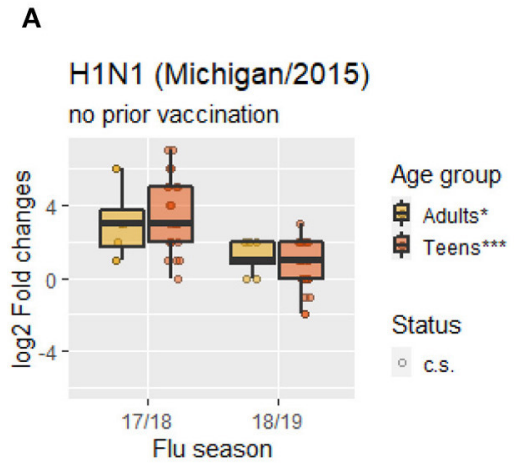

Yamagata-lineage (Phuket/2013) no prior vaccination
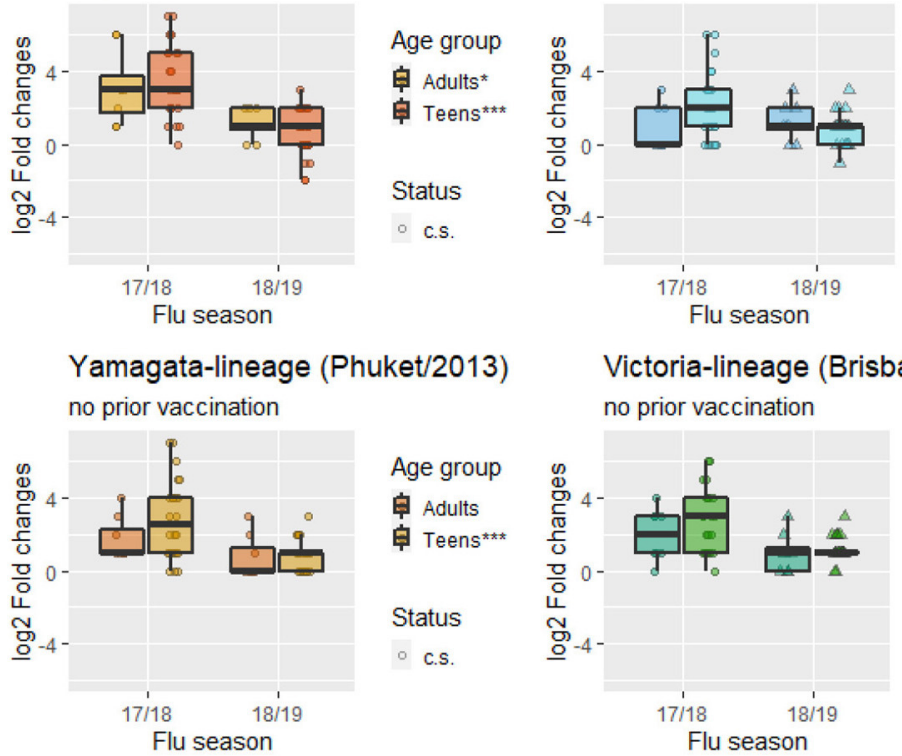

Flu season
Flu season

H3N2 (Hong Kong/2014)

no prior vaccination

Victoria-lineage (Brisbane/2008) no prior vaccination

Age group

追 Adults

自 Teens ${ }^{\star \star \star x}$

Status

- c.s.

$\Delta$ noc.s.

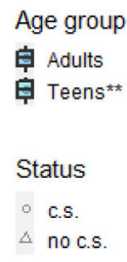

and

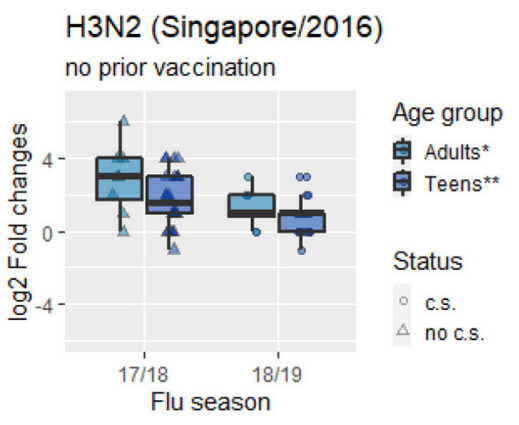

Victoria-lineage (Colorado/2017) no prior vaccination

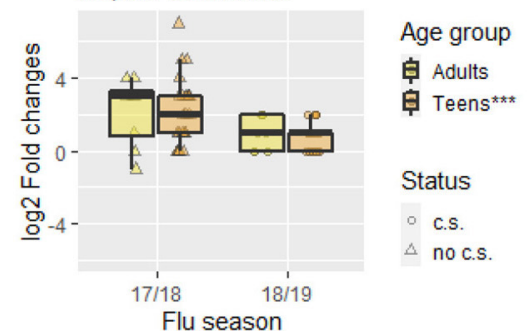

Flu season

B

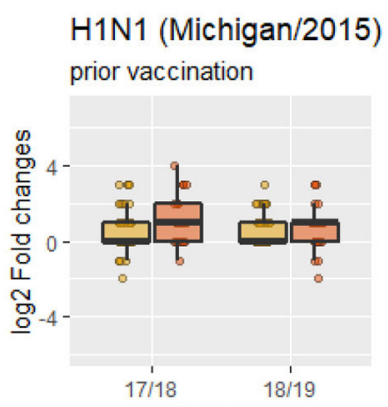

Flu season

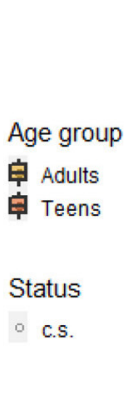

Age group

皁 Adults

Teens

Status

Yamagata-lineage (Phuket/2013)

prior vaccination

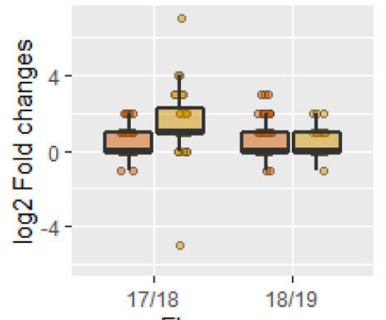

Flu season

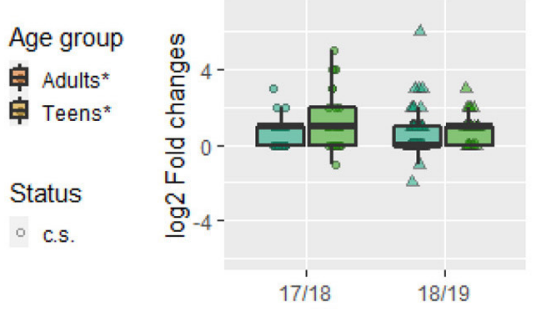

Flu season
H3N2 (Hong Kong/2014)

prior vaccination

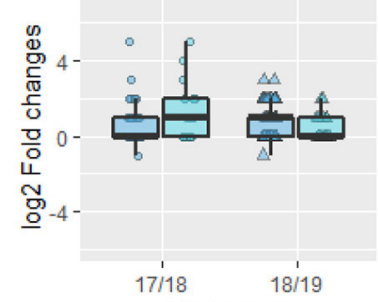

Flu season

Victoria-lineage (Brisbane/2008) prior vaccination

Age group
鼻 Adults
帛 Teens
Status
$\therefore$ c.s.
$\triangle$ noc.s.

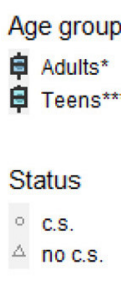

(1)

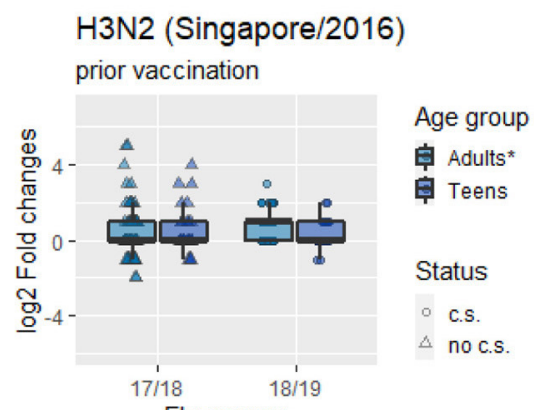

Flu season

Victoria-lineage (Colorado/2017) prior vaccination

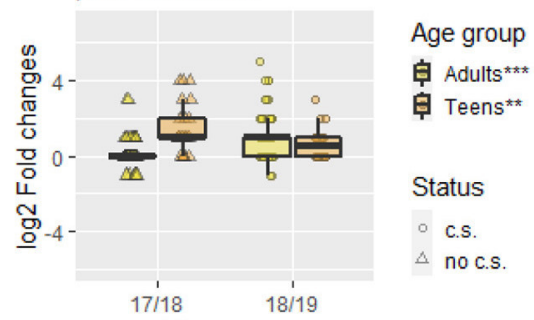

Flu season

FIGURE 4 | Mean folds changes in HAl titers for each vaccine component strain stratified by age groups and prior vaccination status. (A) Subgroup with prior vaccination. (B) Subgroup with no prior vaccination. (c.s. in current season's vaccine formulation; no c.s. not in current season's vaccine formulation; ${ }^{* \star *} p<0.001$, $\left.{ }^{* *} p<0.01,{ }^{*} p<0.05\right)$.

provides the 2018-2019 season descriptive statistics for teenagers repeatedly enrolled in the 2 consecutive seasons and those newly enrolled for the first time in the 2018-2019 season.
While there were $51 \%$ of newly enrolled teenagers reported to be vaccinated in the 2017-2018 flu season, their average HAI composite score was still significantly higher than that 
from the re-enrolled teenagers. A further stratification of the newly enrolled teenagers by their previous year vaccination status suggests that those who were vaccinated in the previous season had a significantly lower mean HAI composite score (4.10 vs. 11.60).

\section{Multivariate Analysis}

In the linear regression analysis for all 140 participants, baseline HAI titers, age, BMI, prior vaccination in 2016-2017, and flu season $18 / 19$ showed significant negative associations with HAI composite scores (Table 2). The negative coefficient for the flu season 18/19 suggested a decline in vaccine-elicited antibody responses between the years. In addition, the interaction term of age and BMI were positively significant. Similar associations between the host factors and HAI composite scores were found in the logistic regression model when the outcome was dichotomized (Supplementary Table 2). In the stratified analyses with different age groups, baseline HAI titers, history of vaccination, and flu season 18/19 significantly correlated with HAI composite scores in both adults and teenagers (Table 2). BMI and the interaction term of age and BMI were only significant in adults. Results from the logistic regression models were generally in consist with the findings from the linear models (Supplementary Table 2).

An additional subgroup analysis stratifying younger and older adults with adjusted models using GEE also suggested the potential negative association in younger adults and positive association in older adults (Supplementary Table 4).

TABLE 2 | Coefficient estimates of host variables fitted in linear model with GEE.

\begin{tabular}{|c|c|c|c|}
\hline & Overall $(n=140)$ & Adults $(n=86)$ & Teens $(n=54)$ \\
\hline $\begin{array}{l}\text { Baseline } \mathrm{HAl} \\
\text { titers }\end{array}$ & $\begin{array}{c}-2.33^{\star \star \star} \\
(-2.79,-1.88)\end{array}$ & $\begin{array}{c}-1.02^{\star \star \star} \\
(-1.32,-0.72)\end{array}$ & $\begin{array}{c}-3.20^{\star \star \star} \\
(-3.84,-2.57)\end{array}$ \\
\hline Age & $\begin{array}{c}-0.21^{\star \star} \\
(-0.34,-0.08)\end{array}$ & $\begin{array}{c}-0.15^{\star} \\
(-0.27,-0.02)\end{array}$ & $\begin{array}{c}0.82 \\
(-2.22,3.87)\end{array}$ \\
\hline Sex (Male) & $\begin{array}{c}-0.08 \\
(-1.50,1.35)\end{array}$ & $\begin{array}{c}0.29 \\
(-1.19,1.77)\end{array}$ & $\begin{array}{c}-0.44 \\
(-15.11,14.23)\end{array}$ \\
\hline Race (White) & $\begin{array}{c}0.64 \\
(-0.21,1.49)\end{array}$ & $\begin{array}{c}0.13 \\
(-0.61,0.86)\end{array}$ & $\begin{array}{c}1.14 \\
(-0.27,2.55)\end{array}$ \\
\hline BMI & $\begin{array}{c}-0.25^{\star \star} \\
(-0.41,-0.09)\end{array}$ & $\begin{array}{c}-0.23^{\star} \\
(-0.40,-0.05)\end{array}$ & $\begin{array}{c}0.18 \\
(-1.69,2.06)\end{array}$ \\
\hline $\begin{array}{l}\text { Comorbidity } \\
\text { (Yes) }\end{array}$ & $\begin{array}{c}-0.32 \\
(-1.14,0.51)\end{array}$ & $\begin{array}{c}-0.38 \\
(-1.19,0.43)\end{array}$ & $\begin{array}{c}0.18 \\
(-1.03,1.39)\end{array}$ \\
\hline $\begin{array}{l}\text { Prior } \\
\text { vaccination } \\
\text { (Yes) }\end{array}$ & $\begin{array}{c}-3.34^{\star \star \star} \\
(-4.22,-2.47)\end{array}$ & $\begin{array}{c}-3.16^{\star \star \star} \\
(-4.62,-1.70)\end{array}$ & $\begin{array}{c}-3.16^{\star \star \star} \\
(-4.50,-1.82)\end{array}$ \\
\hline $\begin{array}{l}\text { Flu season } \\
(18 / 19)\end{array}$ & $\begin{array}{c}-3.15^{\star \star \star} \\
(-3.87,-2.44)\end{array}$ & $\begin{array}{c}-0.83^{\star} \\
(-1.50,-0.16)\end{array}$ & $\begin{array}{c}-4.36^{\star \star \star} \\
(-5.57,-3.16)\end{array}$ \\
\hline $\mathrm{Age}^{*} \mathrm{BMl}$ & $\begin{array}{c}0.005^{\star} \\
(0.0008,0.01)\end{array}$ & $\begin{array}{c}0.005^{\star} \\
(0.0005,0.01)\end{array}$ & $\begin{array}{c}-0.02 \\
(-0.15,0.10)\end{array}$ \\
\hline $\begin{array}{l}\text { Age }^{*} \text { Sex } \\
\text { (Male) }\end{array}$ & $\begin{array}{c}-0.003 \\
(-0.05,0.04)\end{array}$ & $\begin{array}{c}-0.02 \\
(-0.06,0.02)\end{array}$ & $\begin{array}{c}-0.005 \\
(-1.02,1.01)\end{array}$ \\
\hline
\end{tabular}

95\% confidence interval in parentheses, ${ }^{* * *} p<0.001,{ }^{* *} p<0.01,{ }^{*} p<0.05$. The bold values are highlight model parameter estimates that are statistically significant.
However, due to the limited sample size in each subgroup, these estimates did not reach statistical significance at the 0.05 level.

\section{DISCUSSION}

Using an HAI composite score as the targeted outcome, we applied GLMs with GEE to assess the associations between vaccine-elicited immune responses and host factors, both repeatedly measured in a human cohort of volunteers who were vaccinated in 2017-2018 and 2018-2019. Overall, HAI composite scores declined significantly from year 1 to year 2 in teenagers but remained steady in adults, likely due to a much higher proportion of adults already receiving vaccination in the previous season. Adults who received vaccination in the previous season had an increase in antibody responses after repeated vaccination, suggesting the benefit of annual vaccination. However, teenagers tend to have lower antibody boosts regardless of their previous vaccination status, implying that a different vaccination strategy might be more beneficial for them. A comparison of mean HAI composite scores in 20182019 between the repeatedly enrolled teenagers and those newly enrolled in 2018-2019 also supports the hypothesis that repeated vaccination resulted in reduced immune responses in teenagers (Supplementary Table 5).

In terms of subtype of influenza virus, the mean fold changes in HAI titers between pre- and post-vaccination dropped significantly in the second flu season for those strains included in both years' vaccine formula (H1N1, B/Yamagata), suggesting a repeated vaccination with the same $\mathrm{H} 1 \mathrm{~N} 1$ and $\mathrm{B} /$ Yamagata strains was associated with reduced boosting of immune responses. Mean fold changes to the $\mathrm{H} 3 \mathrm{~N} 2$ vaccine strains remained at a moderate level without significant differences between the 2 years. The A/Hong Kong/2014 strain included in 2017-2018 was also included in the prior 2016-2017 season but replaced by A/Singapore/2016 in 2018-2019. For B/Victoria, a reduced boost was observed for the B/Brisbane/2008 after it was replaced by B/ Colorado/2017 in the second year, suggesting a lower level of cross-protective immune responses for B/Brisbane/2008. Slight increases were observed in adults with prior vaccination in 4 strains, while declines were observed across all strains among teenagers regardless of their previous year vaccination status. Vaccine-elicited antibody responses to repeated inoculations appear to be quite different between adults and teenagers. We believe repeated vaccination at least partially explains for the decline in HAI composite scores for the H1N1 and $\mathrm{B} /$ Phuket strain that were included in both vaccines. Based on the antigenic distance hypothesis, vaccine effectiveness tends to be low when the past and current vaccine strains are similar $(24,25)$. Some recent studies have also observed reduced vaccine effectiveness in repeat vaccines $(25,26)$. Meanwhile, there is no change (nor elevation) in the strains that were changed between the two seasons, as those strains were not repeatedly included in the vaccine formula, and thus not subjected to the same level of reduced effectiveness. 
There was no statistical difference in HAI composite scores for H3N2/Singapore and B/Victoria/Colorado between 20172018 and 2018-2019. However, Figures 4A,B revealed that this might be due to a mixture pattern of those who were vaccinated in the 2016-2017 season vs. those not vaccinated, i.e., adults who were vaccinated in 2016-2017 tend to have deficient crossreactivities in 2017-2018, but then in 2018-2019 their responses were higher after vaccination (Figure 4A). Meanwhile, both adults and teenagers who were not vaccinated in 2016-2017 had relatively high cross-reactivities for H3N2/Singapore and B/Victoria/Colorado in 2017-2018, but then in 2018-2019 their responses were lower after vaccination (Figure 4B). In addition, a recent study suggested that $\mathrm{H} 1$ virus or type B influenza pre-exposure has a negative correlation with the H3-specific post-vaccination response (27). Specifically, prior exposure to H1 virus may significantly affect cross-reactivities of antibody responses elicited by $\mathrm{H} 3$ virus as long as the $\mathrm{H} 1$ virus is in circulation. This finding may help explain the low HAI responses of H3N2/Singapore in adults with prior vaccination in the 20172018 flu season.

In the general cohort, our findings suggest that baseline HAI titers, age, BMI, prior vaccination in 2016-2017, and flu season were significantly associated with the repeatedly measured HAI composite scores in consecutive years. All five variables were negatively associated with the HAI composite scores, indicating lower vaccine-elicited antibody responses in participants who had higher baseline titers, were older, with higher BMIs, or received flu shots in the previous flu season. There was also an overall decline in the 2018-2019 season after other variables were adjusted. The negative impacts of high baseline antibody levels and previous vaccination have been established in the literature $(3,6-8,28)$. The negative aging effect on immune response found in our study is also consistent with earlier studies showing that antibody responses to influenza vaccines in the elderly were less likely to reach seroprotection and seroconversion than young adults (8). In addition, a lower rate of seroprotection in the elderly was observed against influenza A and B with repeated vaccinations $(29,30)$.

Obesity has been considered a risk factor of influenza infection, possibly through impairing human immune response as well as the effectiveness of vaccination $(14,31)$. The risk of developing influenza and influenza-like illness were twice in vaccinated obese subjects compared with healthy weight subjects (18). Pathological mechanisms related to obesity could have delayed and blunted antiviral responses to influenza virus infection that may increase the risk of severe disease $(16,32)$. Serological studies showed that H1N1 vaccines could induce higher initial serologic responses among obese adults, but greater declines in their antibody titers were observed in the follow-up (14-16). However, no studies were conducted to establish the association between BMI and the overall vaccineelicited antibody responses in a repeated vaccination setting, and its interactive effect with age has not been well-studied. In our analysis, the association between BMI and the repeatedly measured overall induced immune responses differed with age. BMI was not identified as a risk factor for teenagers in our study. We did, however, find that BMI plays an important role in adults, and observed different patterns for young and older adults. Generally, younger adults with higher BMIs tend to have fewer folds' increases in total HAI responses than those with low or normal BMIs, but the association reversed for the elderly, with a turning point around the age of 50. In study participants 50 years and above, higher BMIs were associated with increasing folds of change in HAI titers after vaccination. However, a recent study illustrated that the risk of having influenza in relation to BMI is similar when comparing middle-aged and older adults (31).

Subsequent analyses were conducted stratifying the study population by adults and teenagers. Prior vaccination remained a significant predictor for lower HAI scores in both groups, suggesting that those who had flu shots in the previous season, regardless of their ages, are less likely to respond well to annual flu vaccinations in the following seasons. This finding is consistent with previous studies indicating that prior vaccination status significantly influenced the HAI titers levels from postvaccination $(3,4)$. The interactive effect between age and BMI was only observed in adults. This is perhaps due to the limited sample size and the lack of diversities in both age and BMI values among the teenagers. The age range for the teenagers was between 12 and 17 years old, and their BMI values were mostly at the normal level, with very few exceptions presenting overweight or obesity. The insufficient heterogeneity of BMI values in the enrolled teenage group prohibited a further investigation on its interaction with age in the logistic regression.

An important strength of the performed analyses is the appropriate adjustment of the correlation among repeated measurements by GEE in a longitudinal analysis. Despite of the many longitudinal studies that assessed the relationships between human immune responses and host factors, very few adjusted for the correlation of repeated antibody measurements from the same subject $(17,18)$, and neither of them used a comprehensive measure to assess the overall induced antibody levels across multiple flu strains. Instead of focusing on the individual HAI titers considering each single strain of influenza virus, an HAI composite score combining multiple strains was adopted in our study to evaluate the overall vaccine-elicited human immune responses. Such an index allows a more flexible assessment of immune responses to repeated vaccinations when one or more components in the vaccine formula change between the years. In addition, the inclusion of data from a cohort of teenagers in our study provides valuable insights into an understudied population.

Our study also has several limitations. The statistical power of the conducted analyses was limited by the number of participants who re-enrolled in our cohort for consecutive flu seasons, although the repeated measurements contributed valuable additional data points for the assessment of annual vaccinations. Our study sample was dominated by white participants. While we made the comparison between white and non-white participants, a further detailed analysis on the more vulnerable racial groups was not performed due to the limited number of subjects in those groups. Our investigation of the understudied teenager population was somewhat restricted by the lack of heterogeneity in BMI and comorbidity among the enrolled sample. In addition, the current study only includes data from 2 consecutive vaccinations, comparing immunity for more 
than two seasons may provide further insights into the analysis. However, including more consecutive years would also further reduce the sample size based on the study focus on subjects with repeated vaccinations in consecutive seasons. For all the aforementioned reasons, future cohort studies with larger sample sizes and longer observation durations are highly demanded. Meanwhile, HAI is just one correlate of protection for influenzas, and others (e.g., neutralizing antibodies or T-cells) also help protect. However, we did not have those data in the current cohort study.

In conclusion, our study found distinct vaccine-elicited immune responses between adults and teenagers when both were repeatedly vaccinated in consecutive years. It also provided further supports for the associations between several host factors and antibody responses in a repeated vaccination setting. Notably, we found an interactive effect of age and BMI in the overall population and the adults. Future studies with a larger enrollment of teenaged participants representing a broader range of BMIs may provide additional insights into the interactive effect in the younger age group.

\section{DATA AVAILABILITY STATEMENT}

The raw data supporting the conclusions of this article will be made available by the authors, without undue reservation.

\section{ETHICS STATEMENT}

The studies involving human participants were reviewed and approved by the Institutional Review Board of the University of

\section{REFERENCES}

1. Hoskins TW, Davies J, Smith AJ, Miller C, Allchin A. Assessment of inactivated influenza-a vaccine after three outbreaks of influenza a at christ's hospital. Lancet. (1979) 313:33-5. doi: 10.1016/S0140-6736(79)90468-9

2. Keitel WA, Cate TR, couch RB. Efficacy of sequential annual vaccination with inactivated influenza virus vaccine1. Am J Epidemiol. (1988) 127:35364. doi: 10.1093/oxfordjournals.aje.a114809

3. Beyer WEP, Palache AM, Sprenger JWM, Hendriksen E, Tukker JJ, Darioli R, et al. Effects of repeated annual influenza vaccination on vaccine sero-response in young and elderly adults. Vaccine. (1996) 14:13319. doi: 10.1016/S0264-410X(96)00058-8

4. Keitel WA, Cate TR, Couch RB, Huggins LL, Hess KR. Efficacy of repeated annual immunization with inactivated influenza virus vaccines over a five year period. Vaccine. (1997) 15:1114-22. doi: 10.1016/S0264-410X(97)00003-0

5. Beyer WEP, de Bruijn IA, Palache AM, Westendorp RGJ, Osterhaus ADME. Protection against influenza after annually repeated vaccination: a metaanalysis of serologic and field studies. Arch Intern Med. (1999) 159:1828. doi: 10.1001/archinte.159.2.182

6. McLean HQ, Thompson MG, Sundaram ME, Meece JK, McClure DL, Friedrich TC, et al. Impact of repeated vaccination on vaccine effectiveness against influenza $\mathrm{A}(\mathrm{H} 3 \mathrm{~N} 2)$ and B during 8 seasons. Clin Infect Dis. (2014) 59:1375-85. doi: 10.1093/cid/ciu680

7. Beyer WEP, Palache AM, Baljet M, Masurel N. Antibody induction by influenza vaccines in the elderly: a review of the literature. Vaccine. (1989) 7:385-94. doi: 10.1016/0264-410X(89)90150-3

8. Goodwin K, Viboud C, Simonsen L. Antibody response to influenza vaccination in the elderly: a quantitative review. Vaccine. (2006) 24:115969. doi: 10.1016/j.vaccine.2005.08.105
Georgia. Written informed consent to participate in this study was provided by the participants' legal guardian/next of kin.

\section{AUTHOR CONTRIBUTIONS}

M-HS: literature search, figures, data analysis, data interpretation, and writing. YS: study design, data analysis, data interpretation, figures, and writing. AH and TR: study design, data interpretation, and writing. JB: data interpretation and writing. All authors contributed to the article and approved the submitted version.

\section{FUNDING}

This study received financial support from University of Georgia Research Foundation. This project has been funded by the National Institute of Allergy and Infectious Diseases, a component of the NIH, Department of Health and Human Services, under contract 75N93019C00052. TR is also supported by the Georgia Research Alliance as an Eminent Scholar. $\mathrm{AH}$ also received partial support from $\mathrm{NIH}$ grant U01AI150747. The funders had no role in the study design, data collection and analysis, decision to publish, or preparation of the manuscript.

\section{SUPPLEMENTARY MATERIAL}

The Supplementary Material for this article can be found online at: https://www.frontiersin.org/articles/10.3389/fimmu. 2021.642791/full\#supplementary-material
9. Mosterín Höpping A, McElhaney J, Fonville JM, Powers DC, Beyer WEP, Smith DJ. The confounded effects of age and exposure history in response to influenza vaccination. Vaccine. (2016) 34:540-6. doi: 10.1016/j.vaccine.2015.11.058

10. Engler RJM, Nelson MR, Klote MM, VanRaden MJ, Huang CY, Cox NJ, et al. Half- vs full-dose trivalent inactivated influenza vaccine (2004-2005): age, dose, and sex effects on immune responses. Arch Intern Med. (2008) 168:2405-14. doi: 10.1001/archinternmed.2008.513

11. Khurana S, Verma N, Talaat KR, Karron RA, Golding H. Immune Response following H1N1pdm09 vaccination: differences in antibody repertoire and avidity in young adults and elderly populations stratified by age and gender. $J$ Infect Dis. (2011) 205:610-20. doi: 10.1093/infdis/jir791

12. Gabriel G, Arck PC. Sex, immunity and influenza. J Infect Dis. (2014) 209:S939. doi: 10.1093/infdis/jiu020

13. Chambers C, Skowronski DM, Rose C, Serres GD, Winter AL, Dickinson JA, et al. Should sex be considered an effect modifier in the evaluation of influenza vaccine effectiveness? Open Forum Infect Dis. (2018) 5:ofy211. doi: 10.1093/ofid/ofy211

14. Sheridan PA, Paich HA, Handy J, Karlsson EA, Hudgens MG, Sammon AB, et al. Obesity is associated with impaired immune response to influenza vaccination in humans. Int J Obes. (2012) 36:1072-7. doi: 10.1038/ijo. 2011.208

15. Callahan ST, Wolff M, Hill HR, Edwards KM, Vaccine N, Treatment Evaluation Unit Pandemic HNVSG. Impact of body mass index on immunogenicity of pandemic $\mathrm{H} 1 \mathrm{~N} 1$ vaccine in children and adults. $J$ infect Dis. (2014) 210:1270-4. doi: 10.1093/infdis/jiu245

16. Honce R, Schultz-Cherry S. Impact of obesity on influenza A virus pathogenesis, immune response, and evolution. Front Immunol. (2019) 10:1071. doi: 10.3389/fimmu.2019.01071 
17. Groenwold $\mathrm{RHH}$, Hoes AW, Hak E. Impact of influenza vaccination on mortality risk among the elderly. Euro Respir J. (2009) 34:5662. doi: 10.1183/09031936.00190008

18. Neidich SD, Green WD, Rebeles J, Karlsson EA, Schultz-Cherry $\mathrm{S}$, Noah TL, et al. Increased risk of influenza among vaccinated adults who are obese. Int J Obes. (2017) 41:1324-30. doi: 10.1038/ijo. 2017.131

19. Petrie JG, Ohmit SE, Johnson E, Cross RT, Monto AS. Efficacy studies of influenza vaccines: effect of end points used and characteristics of vaccine failures. J Infect Dis. (2011) 203:1309-15. doi: 10.1093/infdis/jir015

20. Ohmit SE, Petrie JG, Cross RT, Johnson E, Monto AS. Influenza hemagglutination-inhibition antibody titer as a correlate of vaccine-induced protection. J Infect Dis. (2011) 204:1879-85. doi: 10.1093/infdis/jir661

21. WHO Global Influenza Surveillance Network W. Manual for the Laboratory Diagnosis and Virological Surveillance of Influenza. Geneva: WHO (2011).

22. Abreu RB, Kirchenbaum GA, Clutter EF, Sautto GA, Ross TM. Preexisting subtype immunodominance shapes memory B cell recall response to influenza vaccination. JCI Insight. (2020) 5:e132155. doi: 10.1172/jci.insight.132155

23. R Core Team. R: A Language and Environment for Statistical Computing. Vienna: R Foundation for Statistical Computing (2020).

24. Smith DJ, Forrest S, Ackley DH, Perelson AS. Variable efficacy of repeated annual influenza vaccination. Proc Natl Acad Sci USA. (1999) 96:140016. doi: 10.1073/pnas.96.24.14001

25. Skowronski DM, Chambers C, De Serres G, Sabaiduc S, Winter AL, Dickinson JA, et al. Serial vaccination and the antigenic distance hypothesis: effects on influenza vaccine effectiveness during A(H3N2) epidemics in Canada, 20102011 to 2014-2015. J Infect Dis. (2017) 215:1059-99. doi: 10.1093/infdis/jix074

26. Cobey S, Gouma S, Parkhouse K, Chambers BS, Ertl HC, Schmader KE, et al. Poor immunogenicity, not vaccine strain egg adaptation, may explain the low H3N2 Influenza vaccine effectiveness in 2012-2013. Clin Infect Dis. (2018) 67:327-33. doi: 10.1093/cid/ciy097

27. Xie H, Li L, Ye Z, Li X, Plant EP, Zoueva O, et al. Differential effects of prior influenza exposures on H3N2 cross-reactivity of human postvaccination sera. Clin Infect Dis. (2017) 65:259-67. doi: 10.1093/cid/cix269
28. Thompson MG, Naleway A, Fry AM, Ball S, Spencer SM, Reynolds S, et al. Effects of repeated annual inactivated influenza vaccination among healthcare personnel on serum hemagglutinin inhibition antibody response to A/Perth/16/2009 (H3N2)-like virus during 2010-11. Vaccine. (2016) 34:9818. doi: 10.1016/j.vaccine.2015.10.119

29. Carlock MA, Ingram JG, Clutter EF, Cecil NC, Ramgopal M, Zimmerman RK, et al. Impact of age and pre-existing immunity on the induction of human antibody responses against influenza B viruses. Hum Vaccin Immunother. (2019) 15:2030-43. doi: 10.1080/21645515.2019.1642056

30. Nuñez IA, Carlock MA, Allen JD, Owino SO, Moehling KK, Nowalk $\mathrm{P}$, et al. Impact of age and pre-existing influenza immune responses in humans receiving split inactivated influenza vaccine on the induction of the breadth of antibodies to influenza A strains. PLoS ONE. (2017) 12:e0185666. doi: 10.1371/journal.pone.0185666

31. Karki S, Muscatello DJ, Banks E, MacIntyre CR, McIntyre P, Liu B. Association between body mass index and laboratory-confirmed influenza in middle aged and older adults: a prospective cohort study. Int J Obes. (2018) 42:14808. doi: 10.1038/s41366-018-0029-x

32. Paich HA, Sheridan PA, Handy J, Karlsson EA, Schultz-Cherry S, Hudgens MG, et al. Overweight and obese adult humans have a defective cellular immune response to pandemic H1N1 influenza A virus. Obesity. (2013) 21:2377-86. doi: 10.1002/oby.20383

Conflict of Interest: The authors declare that the research was conducted in the absence of any commercial or financial relationships that could be construed as a potential conflict of interest.

Copyright (C) 2021 Sung, Shen, Handel, Bahl and Ross. This is an open-access article distributed under the terms of the Creative Commons Attribution License (CC BY).

The use, distribution or reproduction in other forums is permitted, provided the original author(s) and the copyright owner(s) are credited and that the original publication in this journal is cited, in accordance with accepted academic practice. No use, distribution or reproduction is permitted which does not comply with these terms. 